\title{
Latencies to name one of three stimulus dimensions: A study of probability effects and dimension integrality
}

\author{
E. SCOTT GELLER \\ Virginia Polytechnic Institute and State University, Blacksburg, Virginia 24061
}

\begin{abstract}
Following each of 400 stimuli that varied according to three binary dimensions (location, color, and shape), subjects named the value of one particular dimension as quickly as possible. Each stimulus appeared on the "left" and "right" side of a screen (location dimension), and was a "red" or "green" triangle (color dimension) with apex oriented "up" or "down" (shape dimension). The alternatives of each dimension varied according to different probability distributions $(90 / 10,70 / 30$, or $50 / 50)$, and three schedule conditions were contrived so that each distribution occurred once for each dimension. Nine groups of 10 subjects each defined the factorial of 3 (response conditions: identify location, color, or shape) by 3 (schedule conditions: 90 left/70 red/50 down, 70 left $/ 50 \mathrm{red} / 90 \mathrm{down}$, or 50 left/90 red/70 down). Latencies to identify the shape dimension were influenced reliably by the probabilities of both response-relevant and responseirrelevant stimuli. Shape probabilities did not affect latencies to identify location of color, but these latencies were significantly influenced by both color and location probabilities. Results are discussed with reference to the locus of the stimulus probability effect and dimensional integrality.
\end{abstract}

Perhaps the most common finding when measuring latencies between stimulus presentations and identification responses is that choice reaction time (RT) is significantly shorter to more frequently occurring stimuli. The phenomenon has been termed a "stimulus probability effect" (SPE); and although the SPE has been consistently observed, interpretations of the cognitive locus of the phenomenon have not been consistent. Specifically, some RT investigators have claimed that the SPE is largely a responsebias effect, resulting from differential response selection (e.g., Theios, 1973, 1975), whereas others have presumed that variations in stimulus frequency affect primarily the stimulus-encoding stage of information processing (e.g., Pachella, 1974; Pachella \& Miller, 1976). The present RT experiment was partially designed to study the relative importance of stimulus-encoding processes in determining the SPE.

Three general techniques have been used to compare the relative influence of stimulus and response processing in determining the SPE: (1) threestimulus, two-response paradigms, (2) additivefactor designs, and (3) S-R compatibility manipulations. Although a given experiment has often showed

Portions of this paper were presented at the Midwestern Psychological Association meeting, Chicago, 1975. Requests for reprints should be sent to E. Scott Geller, Department of Psychology, Virginia Polytechnic Institute and State University, Blacksburg, Virginia 24061 . one information-processing component to be the more significant determinant of the SPE, the particular component judged to be the prominent factor has varied between experiments. For example, when RT was faster to the more probable of two stimuli identified with the same response, Bertleson and Tisseyre (1966) concluded that stimulus (or perceptual) processing was more important than response selection in determining the SPE. Such was also concluded from the three-stimulus, tworesponse RT studies of Hawkins and Hosking (1969), Hawkins, Thomas, and Drury (1970), and LaBerge, Tweedy, and Ricker (1967). However, with this same paradigm, LaBerge, Legrand, and Hobbie (1969) and LaBerge and Tweedy (1964) found evidence of both stimulus and response determinants of the SPE, and Dillon (1966) showed that the SPE occurred only as a result of response bias.

Applications of Sternberg's additive-factor approach (1969) to defining the locus of the SPE are based on the assumption that an interaction between stimulus probability and a factor with a given cognitive locus of RT influence implies a similar locus for the SPE. Thus, the SPE was presumed to result from differential stimulus encoding when Miller and Pachella (1973) observed interactions between stimulus quality and stimulus probability in a Sternberg item-recognition task and in a standard digit-naming RT task. Further, the lack of an interaction between stimulus probability and response type in a Sternberg task provided evidence that the SPE was not due to 
differential response-selection time (e.g., Krueger, 1970; Miller \& Pachella, 1973). In contrast, Biederman and Stacy (1974) demonstrated an interaction between stimulus probability and response type in a Sternberg task, therefore suggesting the responseselection stage as the locus for the SPE.

The S-R compatibility approach to comparing stimulus and response processing is based on the assumption that if an independent variable influences RT to a greater degree in the task with more incompatible (or unnatural) S-R pairings, then that independent variable influences response processing (cf. Sanders, 1970; Welford, 1960). Although most choice RT experiments of this nature have demonstrated reliable increases in the SPE with decreases in S-R compatibility (e.g., Fitts, Peterson, \& Wolpe, 1963; Kanarick, 1966; Posner, 1966; Sanders, 1970), some investigators have observed no such interaction between probability and compatibility in a two-choice RT task (Hawkins \& Friedin, 1972; Hawkins \& Underhill, 1971), and at least one choice RT study observed a significant decrease in the SPE with a decrease in S-R compatibility (Geller, Whitman, \& Farris, 1972).

Compatibility is usually considered highest when subjects identify simple stimuli by vocalizing their familiar name. Indeed, according to one information processing model, the response-selection state is actually bypassed in compatible naming tasks (Theios, 1973). Thus, the failure to find a significant SPE in digit-naming tasks (Forrin, Kumler, \& Morin, 1966; Theios, 1973) supported Theios' theory that the SPE is due to probabilistic push-down memory stacking in the response-determination stage of information processing. However, Pachella (1974) indicated that the absence of the SPE in digit naming may have been due to different speed-accuracy tradeoffs, as suggested by an inverse relationship between stimulus probability and error rate in Theios' study. In fact, when error rates were relatively low and did not vary appreciably as a function of stimulus probability, digit-naming RT showed a significant SPE (Miller \& Pachella, 1973; Stanovich \& Pachella, 1976).

In the present experiment, stimulus presentations varied according to three binary dimensions (location, color, and shape). The two alternatives of each dimension (i.e., left vs. right location, red vs. green color, up vs. down shape) did not occur equally often; instead, the dimension alternatives varied systematically according to independent probability distributions. Subjects vocalized the familiar name for the value of one particular stimulus dimension, and therefore two dimensions of each stimulus presentation were irrelevant to response differentiation. Comparing naming latencies between the two alternatives of the response-irrelevant dimensions represents an innovative technique for studying the relative influence of perceptual factors in the SPE. Thus, an inverse relationship between naming RT and the probability of a response-irrelevant stimulus would reflect differential stimulus encoding (not differential response selection). Even systematic changes in naming RT as a function of the probability of the response-relevant stimuli would suggest perceptual determinants of the SPE, although it might be argued that naming the location, color, or shape of a three-dimensional stimulus is not as compatible as naming a digit.

The degree of integrality (or separability) between stimulus dimensions is a critical determinant of information processing (cf. Garner, 1970). Empirical definitions of integrality have been most often based on performance in a speeded classification task, wherein subjects are required to sort cards displaying multidimensional stimuli according to one stimulus dimension (Garner, 1970, 1974, 1976). The sorting speed for such a task is compared with speed to sort unidimensional stimulus cards in order to determine facilitative or interference effects of the responseirrelevant dimension(s). When the dimensions are redundant or correlated (i.e., the value of one binary dimension always occurs with one value of a second binary dimension), the degree of integrality is a direct function of the extent that subjects sort the multidimensional cards faster than the unidimensional cards. On the other hand, with orthogonal or uncorrelated dimensions (as in the present study), integrality presumably causes interferences in the sort,ing task; and therefore integrality is defined by the extent to which the unidimensional stimulus cards are sorted faster than the multidimensional cards (Garner, 1970, 1974, 1976). A separable stimulus dimension neither facilitates nor interferes with the speeded classification of another dimension.

Reaction time has been used to study the integrality of multidimensional stimuli in standard two-choice RT tasks (e.g., Biederman \& Checkosky, 1970; Felfoldy, 1974) and in same-different RT tasks, wherein the subject is required to indicate whether two stimulus presentations (varying on one, two, or three dimensions) are identical or different (e.g., Downing \& Grossman, 1970; Egeth, 1966). Dimension integrality is shown if correlated dimensions decrease RT (i.e., facilitation or redundancy gain), or if orthogonal dimensions increase RT (i.e., interference). It has been suggested that stimuli from integral dimensions are processed in parallel, whereas separable dimensions are processed serially (cf. Biederman, 1972; Garner, 1976).

The three stimulus dimensions in the present study (location, color, and shape) should all be discriminable from one another, but, as presented, the location dimension may be most discriminable (or separ- 
able) from the other dimensions. For example, it is reasonable to assume that subjects can identify the location of the stimulus (i.e., left or right) without processing the irrelevant dimensions (color and shape), whereas in order to identify the color or shape dimensions it is necessary to locate the stimulus. Further, the color (red vs. green) should be more discriminable and salient than the shapes (upright vs. inverted triangle), and therefore it was hypothesized that the color dimension would be encoded more often than the shape dimension when both of these dimensions were response-irrelevant. The present study estimated the extent that responseirrelevant stimuli were encoded by comparing the SPE between response-irrelevant dimensions.

\section{METHOD}

\section{Subjects}

The $\mathbf{4 5}$ males and 45 females were students from introductory psychology classes at Virginia Polytechnic Institute and State University, and received voluntary research credit for their participation. Each subject was randomly assigned to one of nine experimental conditions defined by three stimulus-probability schedules and three response conditions, with the restriction that each group of 10 subjects contain an equal number of males and females.

\section{Stimuli}

Each of 400 successive events appeared on the left or right side of a presentation screen, and was a red or green equilateral triangle with its apex pointing $u p$ or down. The two stimulus locations and shapes are depicted in Figure 1. The color dimension was varied by the illumination of three red or green christmas-tree lights positioned within the particular shape-location alternative. The average of three illumination measurements for each stimulus (taken $23 \mathrm{~cm}$ from the presentation screen) was as follows: right/ $\mathrm{red} / \mathrm{down}=1.10 \log \mathrm{fL}, \mathrm{right} / \mathrm{red} / \mathrm{up}=1.10 \log \mathrm{fL}$, left $/$ $\mathrm{red} /$ down $=1.05 \log \mathrm{fL}$, left $/ \mathrm{red} / \mathrm{up}=1.10 \mathrm{log} \mathrm{fL}$, right $/$ green $/$ down $=.85 \log \mathrm{fL}$, right/green $/ \mathrm{up}=.90 \log \mathrm{fL}$, left/ green $/$ down $=.90 \log \mathrm{fL}$, left $/$ green $/ \mathrm{up}=.90 \log \mathrm{fL}$. The average of the brightest portions of each of the four walls, the floor, and the ceiling from the subject's chair was $-1.46 \mathrm{log} \mathrm{fL}$.

Three schedule conditions of 30 subjects each were determined by the three Latin square combinations of three different probability distributions $(90 / 10,70 / 30,50 / 50)$ and three binary stimulus dimensions (location, color, shape). For the $90 / 10$ and $70 / 30$ distributions, the more probable dimension value was the same for each subject; i.e., left for the location dimension, red for color, and down for shape. The sequence of 400 three-dimensional events for each probability schedule was randomly determined by filtering a uniform random-number generator on an IBM 370 computer according to the joint probabilities specified by the product of each dimension probability. Thus, for the schedule 90 left $/ 70 \mathrm{red} / 50$ down, the probability (P) for each of the eight possible location/color/shape combinations was as follows: $\mathrm{P}(\mathrm{left} / \mathrm{red} /$ down $)=.90 \times .70 \times .50=.315, \mathrm{P}(\mathrm{left} / \mathrm{red} / \mathrm{up})$ $=.90 \times .70 \times .50=.315, \mathrm{P}($ left $/$ green $/$ down $)=.90 \times$ $.30 \times .50=.135, \mathrm{P}(\mathrm{left} / \mathrm{green} / \mathrm{up})=.90 \times .30 \times .50=.135$, $\mathbf{P}($ right $/ \mathrm{red} /$ down $)=.10 \times .70 \times .50=.035, \mathrm{P}(\mathrm{right} / \mathrm{red} / \mathrm{up})$ $=.10 \times .70 \times .50=.035, \mathrm{P}($ right $/$ green $/$ down $)=.10 \times .30$ $\times .50=.015$, and $P($ right $/$ green $/$ up $)=.015$. For the two blocks of 200 trials, the relative frequency of each stimulus combination matched the appropriate theoretical probability exactly. Hence, for the $90 \mathrm{left} / 70 \mathrm{red} / 50$ down schedule, each 200 trialblock contained 63 occurrences of left/red/down and three occurrences of right/green/up.

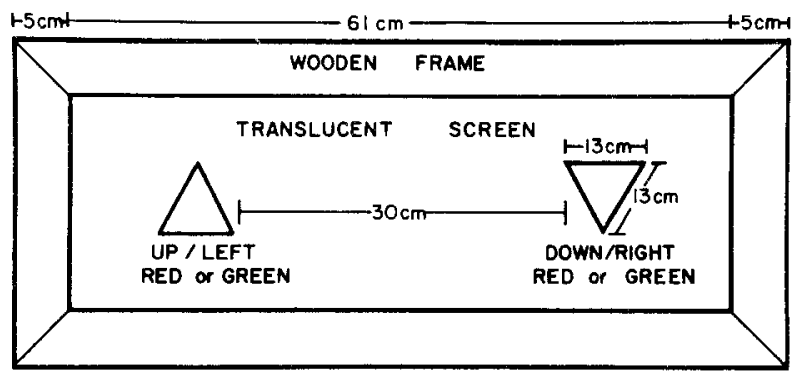

Figure 1. Schematic of the three-dimensional stimulus display. Each stimulus presentation varied according to three binary dimensions: location (left or right), color (red or green), and shape (upright or inverted triangle).

\section{Procedure}

Following the occurrence of each three-dimensional stimulus, subjects verbally identified the alternative of one particular dimension as quickly as possible. A verbal response operated a voice-sensitive relay which turned off the stimulus and stopped a digital-millisecond timer. One group of 30 subjects, 10 subjects for each probability schedule, identified the location of each stimulus presentation (Group Respond/Location); Group Respond/Color $(N=30)$ named the color dimension; and Group Respond/Shape $(\mathbf{N}=30)$ verbalized the shape of each presentation. Thus nine groups of 10 subjects each were determined by the factorial of three probability schedules and three response conditions.

The sequence of events on each trial was as follows: a $.3-\mathrm{sec}$ "ready" buzzer ( $70 \mathrm{~dB}$ SPL), a random time interval ranging from .5 to $1.5 \mathrm{sec}$, a three-dimensional stimulus presentation, the subject's verbal identification of the appropriate dimension alternative, and an intertrial interval ranging from 1.5 to $3 \mathrm{sec}$. The task instructions emphasized the importance of both speed and accuracy in making dimension identifications. Subjects were informed that the sequence was predetermined by a punched paper tape but were given no information concerning the nature of the stimulus sequence. At the beginning of each individual $11 / 2-\mathrm{h}$ session, the subject was given 10 practice trials during which taskrelated questions were answered. Following 200 trials, the subject was given a 5-min break during which he/she was permitted to stand and stretch.

\section{RESULTS}

Inaccurate verbalizations were eliminated from the analysis and did not exceed 15 (i.e., 3.7\%) for any subject. The frequencies of inaccurate verbalizations were studied with analysis of variance (ANOVA) according to the factorial of 3 (response conditions: identify location, color, or shape) by 3 (probability schedules: 90 left/70 red $/ 50$ down, 70 left $/ 50$ red/ 90 down, or 50 left $/ 90 \mathrm{red} / 70$ down). No significant effects were obtained, all ps $>.25$. The average percentages of errors for each response condition were: $.51, .52$, and .59 for Respond/Location, Respond/Color, and Respond/Shape, respectively.

Each choice reaction time (RT) was categorized threefold, each according to one of the three dimension alternatives preceding the verbal reaction. Thus, for the three categorizations of each subject's RTs, one represented the subject's relevant dimension (i.e., the identified dimension), whereas the 
two other categorizations were for the responseirrelevant (nonidentified) dimensions. In other words, for each subject, mean RT was calculated separately for left vs. right, red vs. green, and up vs. down. The mean RTs for each group of 10 subjects are depicted in Figure 2. Since the relative frequency of each three-dimensional combination was determined by the product of the three individual probabilities, a dichotomous classification according to one dimension did not change the frequency bias of the other dimension alternatives. For example, given the schedule $90 \mathrm{left} / 70 \mathrm{red} / 50$ down and a categorization according to the location dimension (left vs. right), $70 \%$ (242) of the 360 left presentations were red colors while $50 \%$ (180) were down shapes. Likewise, of the 40 right presentations of this schedule, 28 , or $70 \%$, were red and 20 , or $50 \%$, were down.

For each classification, a 3 (response condition) by 3 (probability schedule) by 2 (dimension stimulus) ANOVA was performed. Each of these ANOVAs showed a main effect of response, $F(2,81)=10.17$, 9.45 , and 10.92 for the location, color, and shape classifications, respectively ( $\mathrm{ps}<.01$ ), and a $\mathrm{Re}$ sponse by Schedule by Stimulus interaction, $F(4,81)$ $=4.54,3.24$, and 8.31 for the location, color, and shape analyses, respectively (ps $<.025$ ). The response effect is indicated in Figure 2 as shortest RTs to identify location (i.e., the leftmost panel of the figure) and longest RTs to identify shape (the rightmost panel). The pairwise comparisons of response conditions indicated that average RT to name the shape dimension (i.e., the respond/shape condition) was significantly slower than RT to name color and RT to name location (ps $<.01$ ), but the respond/ color RTs were not reliably slower than respond/ location RTs. As depicted in Figure 2, the interactions were due to differential $\mathrm{RT}$ variations as a function of the stimulus alternative of both responserelevant and response-irrelevant dimensions. Probability effects were most prominent for values of the response-relevant dimension, and effects on RT of the response-irrelevant dimensions were most marked when the shape alternatives were identified (the rightmost panel of Figure 2).

The results of planned $t$ tests are summarized in Figure 2, points connected by solid lines being significantly different at the .01 level. Latencies to identify location were not affected by the probability of the shape alternatives, but did vary significantly as a function of both color (response-irrelevant) and location (response-relevant) probabilities. Thus, whenever the red color or left location was more probable (i.e., occurring on $70 \%$ or $90 \%$ of the trials), RT was faster to red than to green colors and faster to left than to right locations.

Latencies to identify colors were shorter to red than to green stimuli for the two schedules with more reds than greens. As for the location-naming RTs, colornaming RT was not influenced by the shape probabilities. The response-irrelevant locations influenced color naming only for the extreme frequency imbalance (i.e., $90 \%$ lefts).

The shape RTs were significantly affected by the response-relevant shape probabilities, as well as by the response-irrelevant locations and colors. That is,

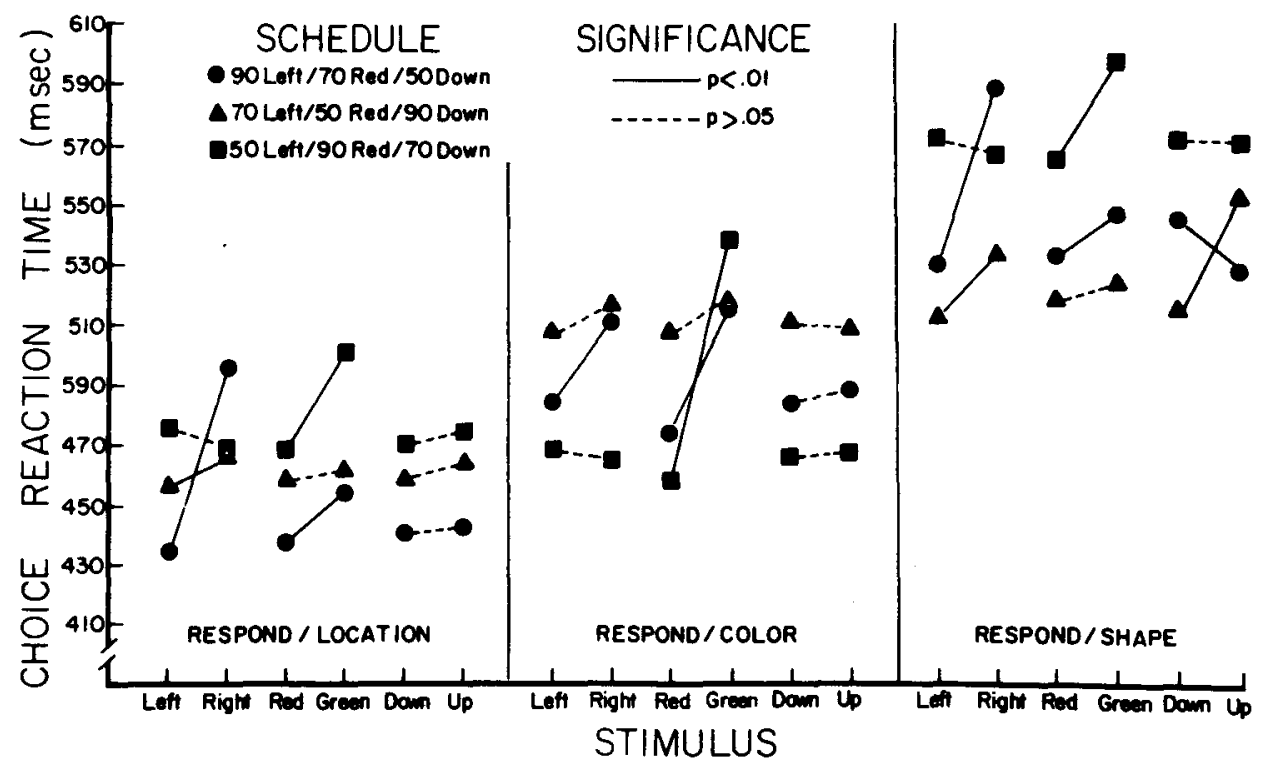

Figure 2. Mean naming RT for each response and schedule condition as a function of three-dimensional dichotomies, one response-relevant and two response-irrelevant. Solid lines connect latency values which are significantly different $(\mathrm{p}<.01)$. 
RT to identify the shape dimension was significantly faster when the more probable location occurred (i.e., $90 \%$ or $70 \%$ lefts) and when the shape was the more probable color (i.e., $90 \%$ or $70 \%$ red). Although shape naming was faster to the more probable down alternative when up and down shapes varied on a $90 / 10$ schedule, shape $R T$ was not faster to down when the frequency imbalance was $70 / 30$. Furthermore, when the two shapes were equiprobable, shape RT was reliably faster to up than to down.

\section{DISCUSSION}

Latencies to name the value of one binary stimulus dimension in a three-dimensional display were reliably influenced by the probabilities of the responserelevant stimuli. Indeed, for each response group, the nature of the stimulus probability effect (SPE) was influenced by the extent of the frequency imbalance. That is, for the respond/location and respond/color conditions, mean RTs to the two dimension alternatives were essentially equivalent when the alternatives were equiprobable; but for both the 70/30 and $90 / 10$ distributions, RT was significantly faster to the more probable alternative, the SPE being prominently greater for the $90 / 10$ than for the $70 / 30$ distribution. Latencies to name the shape dimension were significantly faster to the "up" than to the "down" shape when these alternatives were equiprobable, this difference reflecting a bias due to the relative perceptual familiarity of the stimuli. That is, the "up" shape was a standard triangle, which was a more familiar stimulus than the alternative upsidedown triangle (the "down" shape). This finding is analogous to studies of same-different RT that have demonstrated significantly faster RTs to upright than to inverted numerals (Friden, 1973), trigrams (Egeth \& Blecker, 1971), and letters (Ambler \& Proctor, 1976; Egeth \& Blecker, 1971). In the current study, a 70/30 frequency imbalance that favored the less familiar stimulus neutralized the familiarity effect; and when the less familiar stimulus occurred on $90 \%$ of the trials, naming RT was actually faster to the inverted triangle than the upright triangle.

The significant SPE for each of the responserelevant dimensions is consistent with the findings of some digit-naming, RT studies (Miller \& Pachella, 1973; Stanovich \& Pachella, 1976) but is not consistent with others (Forrin et al., 1966; Theios, 1973). According to Theios' information processing model, the SPE is located in the response-determination stage, and therefore RT to name familiar stimuli with well-learned labels should not vary as a function of stimulus probability, since the time to select a highly compatible response is too negligible to be noticeably affected by probability differences. Clearly nonsupportive of Theios' model are two studies which found a small, but significant, SPE in simple digitnaming tasks (Miller \& Pachella, 1973; Stanovich \& Pachella, 1976). The prominent probability effects for response-relevant stimuli in the present experiment are supportive of a perceptual locus for the SPE. However, it is probable that the stimulusresponse associations were not as natural (or well learned) as in digit naming, and therefore response selection (or "response determination search," to use Theios' term) may have taken sufficient time to change appreciably as a function of stimulus probability. $^{1}$

The observations of the present study that are particularly supportive of a stimulus-encoding locus for the SPE (and thus detrimental to Theios' model) were that, in several cases, naming RT was significantly affected by the probability of a responseirrelevant stimulus. Thus RT to name the shape dimension was faster when the more probable location and color occurred, even though such stimulusdisplay characteristics were completely irrelevant to the response-determination component of information processing. Latencies to identify location and color were not influenced by shape probabilities; but location naming was significantly faster when the more probable color occurred (for both the 70/30 and $90 / 10$ distributions), and stimulus location significantly influenced color naming when the frequency imbalance for location alternatives was extreme (i.e., the $90 / 10$ distribution). Perhaps the response/shape condition resulted in the most consistent response-irrelevant SPEs because stimulus discrimination took longest in this condition, providing time for response-irrelevant perceptual phenomena. Indeed, the relatively slow latencies for the respond/shape condition reflects longer stimulus encoding for shape naming than for either color or location naming, although it is probable that response determination was also longest for the shapenaming condition because, for this condition, the $\mathrm{S}-\mathrm{R}$ associations were least compatible.

Intuitively, it seems that the three stimulus dimensions of the present study were separable from one another (cf. Garner, 1970, 1974), although certain effects of the response-irrelevant stimuli suggest that varying degrees of integrality were represented. For example, the finding that shape identification was influenced by the probability distributions of both the location and color alternatives suggests some degree of integrality between shape and location, and between shape and color. On the other hand, the observation that shape probabilities did not influence either location or color naming indicates that subjects separated (or filtered) the 
location and color dimensions from the shape dimension. These results fit Garner's recent definition of "asymmetric separability," i.e., a dimensional interaction such that "selective attention is possible with orthogonal dimensions, but only with respect to one of the two dimensions" (Garner, 1976, p. 102). Thus, in the present study, location and color were apparently perceived selectively from shape, but shape was not attended to separately from location and color. For example, stimulus location could certainly be identified without processing the shape of the stimulus, but it was necessary to locate the stimulus before identifying the shape dimension. This latter point reflects serial, hierarchial processing that is presumably characteristic of asymmetric separable dimensions (Garner, 1976). Indeed, Garner $(1974$, p. 137) hypothesized that color and shape would be asymmetrically integral in the manner implied by the present findings.

Some degree of integrality between the location and color dimensions is suggested by the findings that response-irrelevant colors affected location naming, and vice versa. Although stimulus location could be identified without attending to the color, the significant effects on location naming of both the $70 / 30$ and $90 / 10$ distributions of color alternatives suggest that some parallel color processing accompanied location naming, perhaps due to the saliency (or discriminability) of the colors. Similarly, the stimulus color could be identified by fixating at a center point between the left and right locations, but stimulus location was apparently encoded in the one schedule condition that presented $90 \%$ left locations. Perhaps, for this condition, the extreme location bias influenced left-directional eye fixations, resulting in faster color identifications when the stimulus appeared on the left. In summary, the differential influence of response-irrelevant stimuli on naming RT to one of three stimulus dimensions indicated particular types of dimensional interactions; i.e., asymmetric separability between color and shape, location and shape, and some degree of integrality between color and location. Furthermore, the nature of a presumed dimensional interaction suggested either serial or parallel information processing that was not inconsistent with intuitive interpretations of the subjects' stimulus perceptions.

\section{REFERENCES}

Ambler, B. A., \& Proctor, J. D. The familiarity effect for singleletter pairs. Journal of Experimental Psychology: Human Perception and Performance, 1976, 2, 222-234.

Bertelson, P., \& Tisse Yre,, R. Choice reaction time as a function of stimulus versus response relative frequency of occurrence. Nature, 1966, 212, 1069-1070.

Biederman. I. Human performance in contingent informationprocessing tasks. Journal of Experimental Psychology, 1972, 93. 219-238.
Biederman, I., \& Checkosky, S. F. Processing redundant information. Journal of Experimental Psychology, 1970, 83, 486-490.

Biederman, I., \& Stacy, E. W. Stimulus probability and stimulus set size in memory scanning. Journal of Experimental Psychology, 1974, 102, 1100-1107.

Dillon, P. J. Stimulus versus response decisions as determinants of the relative frequency effect in disjunctive reaction time performance. Journal of Experimental Psychology, 1966, 71, 321-330.

Downing, B. D., \& Grossman, J. R. Parallel processing of multidimensional stimuli. Perception \& Psychophysics, 1970, 8, 57-60.

Egeth, H. E. Parallel versus serial processes in multidimensional stimulus discrimination. Perception \& Psychophysics, 1966, 1, 245-252.

Egeth, H., \& Blecker, D. Differential effects of familiarity on judgments of sameness and difference. Perception \& Psychophysics, 1971, 9, 321-326.

FELFOLDY, G. L. Repetition effects in choice reaction time to multidimensional stimuli. Perception \& Psychophysics, 1974, 15, 453-459.

Fitrs, P. M., Peterson, J. R., \& Wolpe, G. Cognitive aspects of information processing: II. Adjustments to stimulus redundancy. Journal of Experimental Psychology, 1963, 65, 423-432.

Forrin, B., Kumler, M., \& Morin, R. E. The effects of response code and signal probability in a numeral-naming task. Canadian Journal of Psychology, 1966, 20, 115-124.

FRIDEN, T. P. The effects of familiarity in a perceptual matching task. Perception \& Psychophysics, 1973, 14, 487-492.

GARNER, W. R. Interaction of stimulus dimensions in concept and choice processes. Cognitive Psychology, 1976, 8, 98-123.

GARNER, W. R. The processing of information and structure. Potomac, Md: Erlbaum, 1974.

GARNER, W. R. The stimulus in information processing. American Psychologist, 1970, 25, 350-358.

Geller, E. S., Whttman, C. P., \& Farris, J. C. Probability discrimination indicated by stimulus predictions and reaction speed: Effects of S-R compatibility. Journal of Experimental Psychology, 1972, 93, 404-409.

Hawkins, H. L., \& Friedin, B. D. The relative frequency effect and S-R compatibility. Psychonomic Science, 1972, 28, 229-230.

Hawkins, H. L., \& Hosking, K. Stimulus probability as a determinant of discrete choice reaction time. Journal of Experimental Psychology, 1969, 82, 435-440.

Hawkins, H. L., Thomas, G. B., \& Drury, K. B. Perceptual versus response bias in discrete choice reaction time. Journal of Experimental Psychology, 1970, 84, 514-517.

HawkINS, H. L., \& UNDERHILL, J. R. S-R compatibility and the relative frequency effect in choice reaction time. Journal of Experimental Psychology, 1971, 91, 280-286.

Kanarick, A. F. Probability and payoff as factors influencing twochoice reaction-time. Journal of Engineering Psychology, 1966, 5, 37-46.

KRUEGER, L. E. Effect of stimulus probability on two-choice reaction time. Journal of Experimental Psychology, 1970, 84, 377-379.

LaBerge, D., Legrand, R., \& Hobbie, R. K. Functional identification of perceptual and response biases in choice reaction time. Journal of Experimental Psychology, 1969, 79, 295-299.

LABerge, D.. \& Tweedy, J. R. Presentation probability and choice time. Journal of Experimental Psychology, 1964, 68 , 477-481.

LABerge, D., Tweedy, J. R., \& Ricker, J. Selective attention: Incentive variables and choice time. Psychonomic Science, 1967, 8. $341-342$.

Miller, J. O., \& Pachella, R. G. Locus of the stimulus probability effect. Journal of Experimental Psychology, 1973, 101, 227-231.

Pachella, R. G. The interpretation of reaction time in information processing research. In B. Kantowitz (Ed.), Human information processing: Tutorials in performance and cognition. Hillsdale, N.J: Erlbaum, 1974. 
Pachella, R. G., \& Mirler, J. O. Stimulus probability and samedifferent classification. Perception \& Psychophysics, 1976, 19. 29-34.

Posner, M. L. Components of skilled performance. Science, 1966, 152, 1712-1718.

SANDERS, A. F. Some variables affecting the relation between relative stimulus frequency and choice reaction time. Acta Psychologica, 1970, 33, 45-55.

Stanovich, K. E., \& Pachella, R. G. The effect of stimulus probability on the speed and accuracy of naming alphanumeric stimuli. Bulletin of the Psychonomic Society, 1976, 8, 281-284.

STERNBERG, S. Memory scanning: Mental processes revealed by reaction time experiments. American Scientist, 1969, 57, 421-457.

ThEIOS, J. Reaction time measurements in the study of memory processes: Theory and data. In G. H. Bower (Ed.), The psychology of learning and motivation: Advances in research and theory (Vol. 7). New York: Academic Press, 1973.
Theios, J. The components of response latency in simple human information processing tasks. In P. M. A. Rabbit and S. Dornig (Eds.), Attention and performance $V$. New York: Academic Press, 1975.

WELFORD, A. T. The measurement of sensory-motor performance: Survey and reappraisal of twelve years' progress. Ergonomics, $1960,3,189-229$.

\section{NOTE}

1. The author thanks Dr. Stephen J. Lupker, a former student of Professor Theios, for this response-bias interpretation of the response-relevant SPE.

(Received for publication March 21, 1977; revision accepted May 3, 1977.) 